\title{
Psoriasis-like tinea incognita: a case report and literature review
}

\author{
Laura Đorđević Betetto ${ }^{1}$, Borut Žgavec ${ }^{2}$, Aleksandra Bergant Suhodolčan ${ }^{2,3 凶}$ \\ ${ }^{1}$ Ljubljana Community Health Center, Ljubljana, Slovenia. ${ }^{2}$ Department of Dermatovenereology, Ljubljana University Medical Center, Ljubljana, Slovenia . \\ ${ }^{3}$ Faculty of Medicine, University of Ljubljana, Ljubljana, Slovenia.
}

\begin{abstract}
Tinea incognita is an atypical presentation of fungal infection of the skin, the clinical presentation of which has been modified by misuse of topical corticosteroids or calcineurin inhibitors. Such dermatophyte infections often have an atypical clinical presentation and are difficult to diagnose, but with the rise of immunosuppressive drugs and self-prescribed topical therapies, they are becoming increasingly prevalent. Here we report the case of a 68-year-old male patient with a history of psoriasis, presenting with erythematous scaly lesions, that did not respond to conventional treatment for psoriasis, as would be expected. A diagnosis of tinea incognita was made with histopathological examination with periodic acid-Schiff stain of a skin biopsy sample. This case highlights the fact that dermatophyte infections are widespread but sometimes neglected and can occur concomitantly with other dermatoses.
\end{abstract}

Keywords: tinea incognita, psoriasis, topical steroids, dermatophyte infection, tinea diagnosis, atypical presentation

Received: 16 February 2020| Returned for modification: 5 March 2020|Accepted: 6 March 2020

\section{Introduction}

Superficial dermatophyte infections of the skin are common worldwide and are usually easy to diagnose based on their typical clinical appearance. In recent years, however, there have been increasing reports in the literature about atypical presentations, often with late diagnosis (1-3). Tinea incognito or the grammatically correct term tinea incognita $(4,5)$ is a type of dermatophyte infection of the skin, the clinical presentation of which has been modified by misuse of topical corticosteroids or calcineurin inhibitors (6-8). Tinea incognita can present with various clinical appearances, resembling those of rosacea, eczema, lichen, psoriasis, lupus, viral infections, impetigo, or seborrheic dermatitis $(1,2,9-13)$. It is often recognized in patients with previous skin conditions such as psoriasis that have been treated with topical therapy.

Although the association of some non-dermatological diseases and psoriasis is well established, there are limited data on dermatological comorbidities (14). Of those, fungal infections, especially onychomycosis and tinea pedis, are the ones proven to be more prevalent among patients with psoriasis, often presenting as tinea incognita $(15,16)$. The reason for atypical presentation is most often the use of immunosuppressive drugs and corticosteroids as treatment for psoriasis.

\section{Case report}

A 68-year-old male patient with hypercholesterolemia and arterial hypertension presented to our clinic with aggravation of skin lesions. He reported a longstanding (30-years) history of intermittent exacerbations of mild to moderate psoriasis that had been treated with topical steroids and calcipotriol. This time his clinical examination revealed large erythematous confluent plaques with well-demarcated scaly borders with annular configuration, located on the trunk, buttocks, and lower extremities (Fig. 1). The toenails were hyperkeratotic with pitting. Fungal infection had been ruled out several times by mycological examination with potassium hydroxide $(\mathrm{KOH})$ and culture. Standard treatment for psoriasis with topical steroids and calcipotriol was only partially successful, with rapid recurrence after cessation of the treatment.

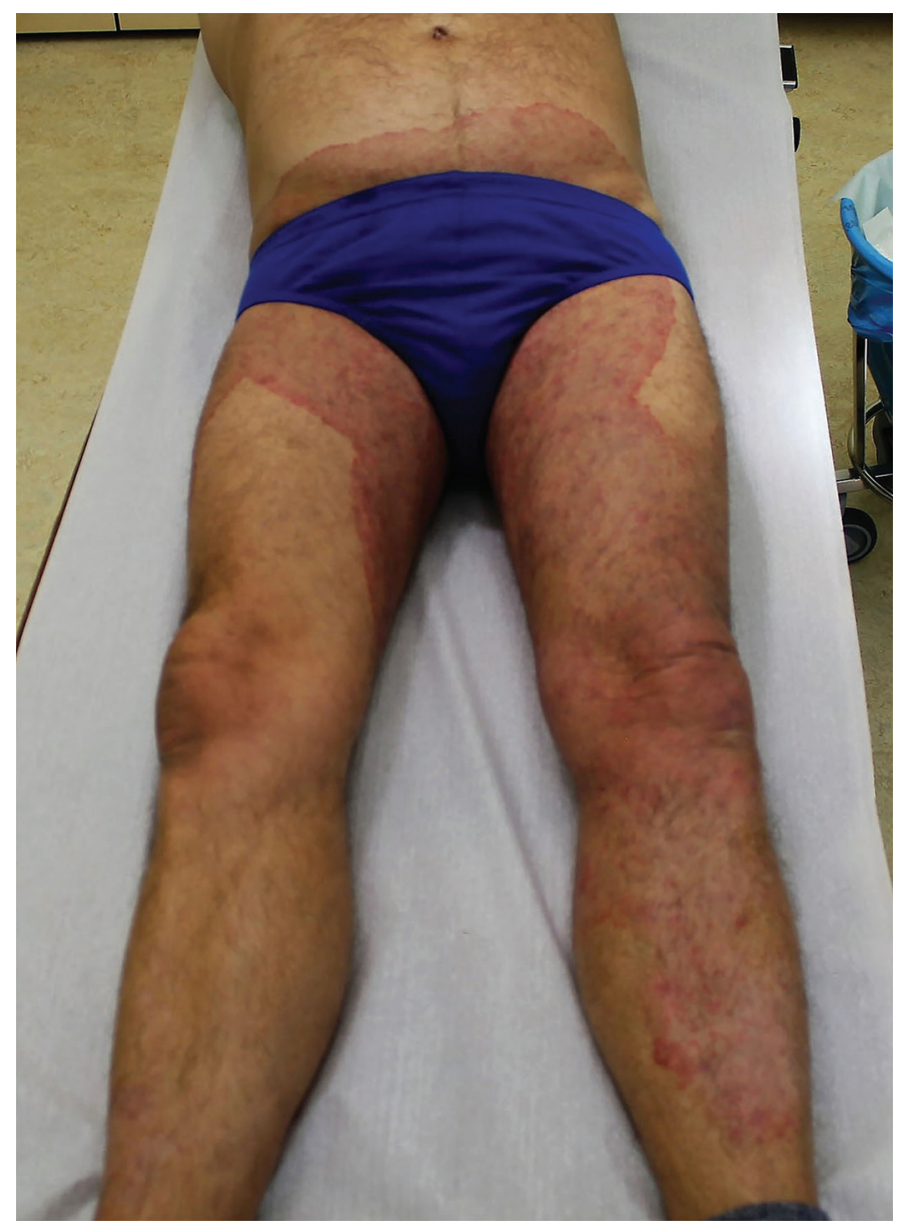

Figure 1| Patient at the time of presentation: large erythematous scaly confluent plaques with well-demarcated borders can be seen on the lower extremities and on the lower part of the trunk. 
Our differential diagnosis included a flare of psoriasis with annular lesions, tinea corporis/tinea incognita, and erythema annulare. Blood tests did not reveal any abnormalities. Mycological examination with $\mathrm{KOH}$ direct microscopy from nail and skin scrapings, taken from the gluteal and interdigital skin and toenails was negative. Skin biopsy from the gluteal region was performed, and the main histopathological feature was suppurative infundibulofolliculitis. The surrounding epidermis showed acanthosis, mild spongiosis, and orthokeratosis with some mounds of parakeratosis and occasional scalecrusts with neutrophils on their summits. The dermis showed mild superficial oedema, sparse perivascular lymphocytic infiltrate, and some neutrophils in the vicinity of folliculitis (Fig. 2). At higher magnification, a few solitary hyphae were noticed in the stratum corneum and were highlighted with periodic acid-Schiff (PAS) stain (Fig. 3), which confirmed a fungal infection.

The patient was treated with systemic and topical terbinafine for 12 weeks. Because the gluteal lesions persisted, we prolonged the systemic treatment to 24 weeks, after which a complete resolution of lesions was achieved.

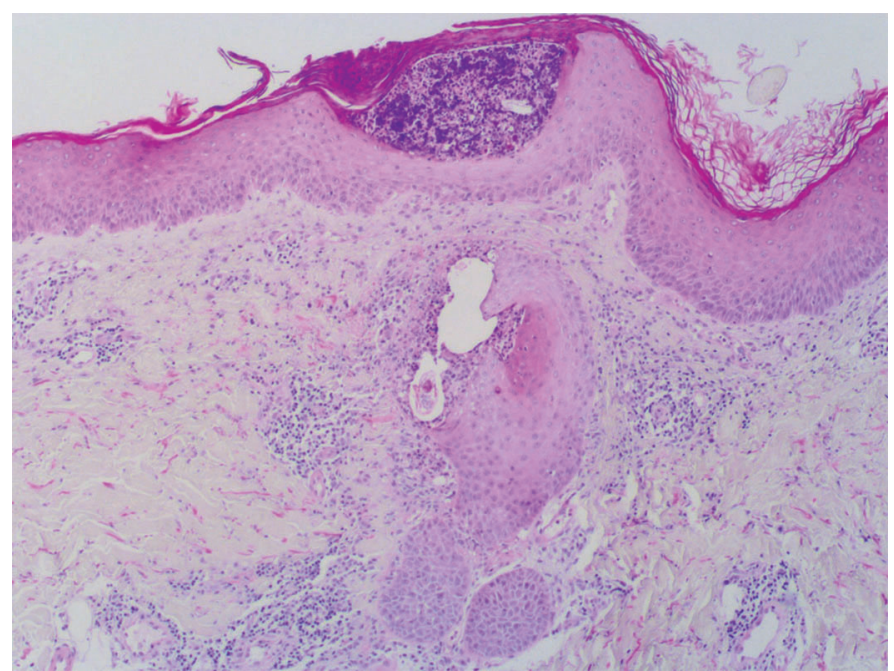

Figure 2 | Hematoxylin and eosin staining (10x magnification): suppurative infundibulofolliculitis with hyperplasia, mild spongiosis, and orthokeratosis in the surrounding epidermis.

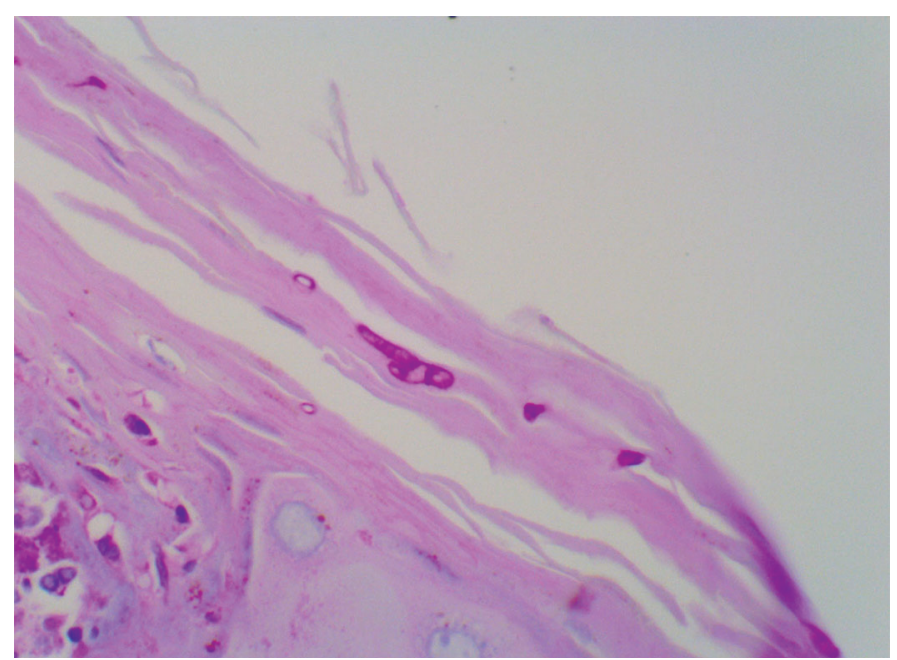

Figure 3 | Periodic acid-Schiff staining (40x magnification): a few solitary hyphae are identified in the stratum corneum.

\section{Discussion}

Immunosuppressive drugs such as corticosteroids, together with other systemic and local factors such as diabetes, HIV infection, and humidity, favour dermatophyte infections of the skin and nails $(17,18)$. When topical corticosteroids and their combinations are applied as a treatment for a pre-existing dermatosis such as psoriasis or as an increasing trend of self-prescribed topical therapy to treat incorrectly self-diagnosed diseases $(19,20)$, they can induce tinea incognita (21).

It is important to note that topical steroids modify the clinical appearance of tinea to some extent, especially reducing inflammatory signs, pruritus, and burning sensation (22), but they do not necessarily make the disease difficult to recognize for an experienced dermatologist (4). The lesions are still asymmetrical, with a defined progression border, and only a minority of cases are rendered unrecognizable (23). In some cases, the clinical appearance of dermatophytosis can be atypical from the beginning even if the patient was never treated with corticosteroids (3). This is due to variations of the pathologic process such as dermatophyte invasive capacity (24), the physiology of the individual, the site of invasion, and/or acquired conditions such as pre-existing skin diseases, sun exposure, and frequent washing (3). Site of involvement predisposes to certain variations, with tinea faciei and tinea corporis having the most misleading presentations (1, 2). Some authors propose that the term "tinea atypica" should be used instead of tinea incognita, in order to include all forms of dermatophytosis that do not have a classical presentation, both from primary and secondary (iatrogenic) factors $(3,25)$.

According to recent literature, tinea atypica is becoming increasingly frequent, with extensive and inflammatory presentations and often late diagnosis $(1-3,25,26)$. This emphasizes the need for an appropriate diagnostic tool. Several studies have shown that histological examination is more sensitive than fungal culture and direct microscopy with $\mathrm{KOH}(27,28)$. In our case, repeated $\mathrm{KOH}$ examination of skin scrapings failed to verify fungal infection, whereas a histopathological analysis with PAS staining confirmed our clinical suspicion. Direct microscopy with or without $\mathrm{KOH}$ offers an immediate result, but for this examination to be adequate, it is essential to obtain a quality sample by trained personnel (29). The downside of histopathological analysis is that it cannot identify the species of dermatophytes because as the only standard method that can do that is culture (28). Because the treatment remains the same regardless of the causative species, this is not a major limitation for clinical practice. In recent years, molecular techniques that employ real-time polymerase chain reaction (PCR) have offered important advances in dermatophyte identification, with an increase in both speed of diagnosis and sensitivity when compared to cultures $(30,31)$. However, the benefits of PCR methods should be balanced against their relatively high costs (30). The best method for diagnosing tinea remains controversial, and some authors still consider clinical examination to be the gold standard" and some authors still consider clinical examination to be the gold standard (32).

In summary, we presented an adult male patient with therapyresistant skin lesions and a history of psoriasis, which, together with negative $\mathrm{KOH}$ and fungal culture, resulted in prolonged treatment with topical corticosteroids. This led to persistent skin lesions that were only diagnosed as tinea incognita when the skin biopsy was performed. Once antifungal treatment was administered, the lesions completely resolved. Dermatophyte infections that occur in patients previously using topical corticosteroids, often have an atypical clinical presentation and are difficult to diagnose, but with the rise of immunosuppressive drugs and self- 
prescribed topical therapies, they are becoming increasingly prevalent. Therefore, we recommend that additional tests, including mycological examination and skin biopsy with histopathology,

\section{References}

1. Ansar A, Farshchian M, Nazeri H, Ghiasian SA. Clinico-epidemiological and mycological aspects of tinea incognito in Iran: a 16-year study. Japanese J Med Mycol. 2011;52:25-32.

2. Kim WJ, Kim TW, Mun JH, Song M, Kim HS, Ko HC, et al. Tinea incognito in Korea and its risk factors: nine-year multicenter survey. J Korean Med Sci. 2013;28: 145-51.

3. Atzori L, Pau M, Aste N, Aste N. Dermatophyte infections mimicking other skin diseases: a 154-person case survey of tinea atypica in the district of Cagliari (Italy). Int J Dermatol. 2012;51:410-5.

4. Verma SB . A closer look at the term "tinea incognito": a factual as well as grammatical inaccuracy. Indian J Dermatol. 2017;62:219-20.

5. Holubar K, Male O. Tinea incognita vs. tinea incognito. Acta Dermatovenerol Croat. 2002;10:39.

6. Crawford KM, Bostrom P, Russ B, Boyd J. Pimecrolimus-induced tinea incognito. Skinmed. 2004:3:352-3.

7. Siddaiah N, Erickson Q, Miller G, Elston DM. Tacrolimus-induced tinea incognito. Cutis. 2004;73:237-8.

8. Rallis E, Koumantaki-Mathioudaki E. Pimecrolimus induced tinea incognito masquerading as intertriginous psoriasis. Mycoses. 2008;51:71-3.

9. Aste N, Pau M, Aste N, Atzori L. Tinea corporis mimicking herpes zoster. Mycoses. 2011;54:463-5.

10. Nicola A, Laura A, Natalia A, Monica P. A 20-year survey of tinea faciei. Mycoses. 2010;53:504-8.

11. Gorani A, Schiera A, Oriani A. Case report. Rosacea-like Tinea incognito. Mycoses. 2002;45:135-7.

12. Serarslan G. Pustular psoriasis-like tinea incognito due to Trichophyton rubrum. Mycoses. 2007;50:523-4.

13. Meymandi S, Wiseman MC, Crawford RI. Tinea faciei mimicking cutaneous lupus erythematosus: a histopathologic case report. J Am Acad Dermatol. 2003;48: S7-8

14. Zander N, Schäfer I, Radtke M, Jacobi A, Heigel H, Augustin M. Dermatological comorbidity in psoriasis: results from a large-scale cohort of employees. Arch Dermatol Res. 2017;309:349-356.

15. Klaassen KMG, Dulak MG, Kerkhof PCM van de, Pasch MC. The prevalence of onychomycosis in psoriatic patients : a systematic review. J Eur Acad Dermatol Venereol. 2014;28:533-41.

16. Alteras I, Ingberg A, Segal R, Schvili D. The incidence of skin manifestations by dermatophytes in patients with psoriasis. Mycopathologia. 1986;95:37-9.

17. Nenoff P, Krüger C, Ginter-Hanselmayer G, Tietz HJ. Mycology-an update. Part 1: Dermatomycoses: causative agents, epidemiology and pathogenesis. JDDG: Journal der Deutschen Dermatologischen Gesellschaft. 2014;12:188-210. be considered when encountering new and/or atypical presentations of pre-existing skin disease.
18. Kaur R, Kashyap B, Bhalla P. Onychomycosis - epidemiology, diagnosis and management. Indian J Med Microbiol. 2008;26:108-16.

19. Bishnoi A, Vinay K, Dogra S. Emergence of recalcitrant dermatophytosis in India. Lancet Infect Dis. 2018;18:250-1.

20. Verma SB, Vasani R. Male genital dermatophytosis - clinical features and the effects of the misuse of topical steroids and steroid combinations - an alarming problem in India. Mycoses. 2016;59:606-14.

21. Ive FA, Marks R. Tinea incognito. BMJ. 1968;3:149-52.

22. van Zuuren EJ, Fedorowicz Z, El-Gohary M. Evidence-based topical treatments for tinea cruris and tinea corporis : a summary of a Cochrane systematic review. $\mathrm{Br}$ J Dermatol. 2015;172:616-41.

23. Atzori L. Tinea infections: changing face or neglected? Am J Biomed Sci Res. 2019;4:298-304.

24. Shiraki Ogawa Y. Role of cytokine secretion of human keratinocytes in dermatophytosis. Nippon Ishinkin Gakkai Zasshi. 2010;51:125-30.

25. Zisova LG, Dobrev HP, Tchernev G, Semkova K, Aliman AA, Chorleva KI, et al. Tin ea atypica: report of nine cases. Wiener Medizinische Wochenschrift. 2013;163: 549-55.

26. Romano C, Maritati E, Gianni C. Tinea incognito in Italy: a 15-year survey. Mycoses. 2006;49:383-7.

27. Weinberg JM, Koestenblatt EK, Tutrone WD, Tishler HR, Najarian L. Comparison of diagnostic methods in the evaluation of onychomycosis. J Am Acad Dermatol. 2003;49:193-7.

28. Karimzadegan-Nia M, Mir-Amin-Mohammadi A, Bouzari N, Firooz A. Comparison of direct smear, culture and histology for the diagnosis of onychomycosis. Australas J Dermatol. 2007;48:18-21.

29. Ingen-Housz-Oro S, Foulet F, Chosidow O. Atypical psoriasis. BMJ. 2015;351: h5510.

30. Sharma R, Gupta S, Asati DP, Karuna T, Purwar S, Biswas D. A pilot study for the evaluation of PCR as a diagnostic tool in patients with suspected dermatophytoses. Indian Dermatol Online J. 2017;8:176-80.

31. Verrier J, Monod M. Diagnosis of Dermatophytosis Using Molecular Biology. Mycopathologia. 2017;182:193-202.

32. Levitt JO, Levitt BH, Akhavan A, Yanofsky H. The sensitivity and specificity of potassium hydroxide smear and fungal culture relative to clinical assessment in the evaluation of tinea pedis: a pooled analysis. Burkhart CG, editor. Dermatol Res Pract. 2010;2010:764843. 